\title{
Mycobacterium leprae-Specific Antibodies in Multibacillary Leprosy Patients Decrease During and After Treatment With Either the Regular 12 Doses Multidrug Therapy (MDT) or the Uniform 6 Doses MDT
}

\section{OPEN ACCESS}

Edited by: Annemieke Geluk, Leiden University Medical Center, Netherlands

Reviewed by: Roberta Olmo Pinheiro, Fundação Oswaldo Cruz

(Fiocruz), Brazil

Anouk Van Hooij, Leiden University Medical Center, Netherlands

*Correspondence:

Mariane M. A. Stefani

mmastefani@gmail.com

tPresent address: Emerith M. Hungria,

Centro Universitário de Anápolis - UniEVANGÉLICA,

Anápolis, Goiás, Brazil

${ }^{ \pm}$Share the senior authorship.

Specialty section: This article was submitted to

Microbial Immunology, a section of the journal

Frontiers in Immunology

Received: 08 November 2017 Accepted: 12 April 2018

Published: 14 May 2018

Citation: Hungria EM, Bührer-Sékula $S$,

Oliveira RM, Aderaldo LC,

Pontes MAA, Cruz R,

Gonçalves HSd, Penna MLF, Penna GO and Stefani MMA (2018)

Mycobacterium leprae-Specific Antibodies in Multibacillary Leprosy Patients Decrease During and After Treatment With Either the Regular 12 Doses Multidrug Therapy (MDT) or the Uniform 6 Doses MDT.

Front. Immunol. 9:915. doi: 10.3389/fimmu.2018.00915

\author{
Emerith M. Hungria ${ }^{1 \dagger}$, Samira Bührer-Sékula', Regiane M. Oliveira', Lúcio C. Aderaldo², \\ Maria Araci A. Pontes ${ }^{2}$, Rossilene Cruz ${ }^{3}$, Heitor S. de Gonçalves ${ }^{2}$, Maria L. F. Penna ${ }^{4}$, \\ Gerson O. Penna ${ }^{5 \neq}$ and Mariane M. A. Stefani ${ }^{1 * \neq}$

\begin{abstract}
Instituto de Patologia Tropical e Saúde Pública, Universidade Federal de Goiás, Goiânia, Brazil, ${ }^{2}$ Centro de Dermatologia ${ }^{4}$ Departamento de Epidemiologia e Bioestatística, Universidade Federal Fluminense, Rio de Janeiro, Brazil, ${ }^{5}$ Núcleo de Medicina Tropical, Universidade de Brasilia, e FIOCRUZ Brasília, Brasilia, Brazil
\end{abstract} \\ Dona Libânia, Fortaleza, Brazil, ${ }^{3}$ Fundação de Dermatologia Tropical e Venereologia Alfredo da Matta, Manaus, Brazil,
}

Leprosy serology reflects the bacillary load of patients and multidrug therapy (MDT) reduces Mycobacterium leprae-specific antibody titers of multibacillary (MB) patients. The Clinical Trial for Uniform Multidrug Therapy Regimen for Leprosy Patients in Brazil (U-MDT/CT-BR) compared outcomes of regular 12 doses MDT/R-MDT and the uniform 6 doses MDT/U-MDT for MB leprosy, both of regimens including rifampicin, clofazimine, and dapsone. This study investigated the impact of R-MDT and U-MDT and the kinetic of antibody responses to $M$. leprae-specific antigens in MB patients from the U-MDT/ CT-BR. We tested 3,400 serum samples from 263 MB patients (R-MDT:121; U-MDT:142) recruited at two Brazilian reference centers (Dona Libânia, Fortaleza, Ceará; Alfredo da Matta Foundation, Manaus, Amazonas). Enzyme-linked immunosorbent assays with three $M$. leprae antigens [NT-P-BSA: trisaccharide-phenyl of phenollic glycolipid-I antigen (PGL-I); LID-1: Leprosy Infectious Disease Research Institute Diagnostic 1 di-fusion recombinant protein; and ND-O-LID: fusion complex of disaccharide-octyl of PGL-I and LID-1] were performed using around 13 samples per patient. Samples were collected at baseline/M0, during MDT (R-MDT:M1-M12 months, U-MDT:M1-M6 months) and after MDT discontinuation (first, second year). Statistical significance was assessed by the Mann-Whitney $U$ test for comparison between groups ( $p$ values $<0.05$ ). Mixed effect multilevel regression analyses were used to investigate intraindividual serological changes overtime. In R-MDT and U-MDT groups, males predominated, median age was 41 and 40.5 years, most patients were borderline lepromatous and lepromatous leprosy (R-MDT:88\%, U-MDT: 90\%). The bacilloscopic index at diagnosis was similar (medians: 3.6 in the R-MDT and 3.8 in the U-MDT group). In R-MDT and U-MDT groups, a significant decline in anti-PGL-I positivity was observed from M0 to M5 $(p=0.035, p=0.04$, respectively), from M6 to M12 and at the first and second year posttreatment $(p<0.05)$. Anti-LID-1 antibodies declined from M0 to M6 ( $p=0.024), M 7$ to M12 in the R-MDT; from M0 to M4 ( $p=0.003)$, M5 to M12 in the U-MDT and posttreatment in both groups $(p>0.0001)$. Anti-ND-O-LID antibodies decreased during and after treatment in both 
groups, similarly to anti-PGL-I antibodies. Intraindividual serology results in R-MDT and U-MDT patients showed that the difference in serology decay to all three antigens was dependent upon time only. Our serology findings in MB leprosy show that regardless of the duration of the U-MDT and R-MDT, both of them reduce $M$. leprae-specific antibodies during and after treatment. In leprosy, antibody levels are considered a surrogate marker of the bacillary load; therefore, our serological results suggest that shorter U-MDT is also effective in reducing the patients' bacillary burden similarly to R-MDT.

\section{Clinical Trial Registration: ClinicalTrials.gov, NCT00669643.}

\section{Keywords: leprosy, serology, phenollic glycolipid-I antigen, LID-1, ND-O-LID, multidrug therapy}

\section{INTRODUCTION}

The infection by Mycobacterium leprae in humans is characterized by a wide spectrum of clinico-pathological manifestations associated with distinct bacteriologic, immunologic, and histopathologic features categorized as tuberculoid (TT), borderline tuberculoid (BT), borderline borderline (BB), borderline lepromatous (BL), and lepromatous leprosy ( $\mathrm{LL})(1)$. In leprosy patients, the specific antibody responses depend on the bacillary load. Vigorous antibody production with low or absent $M$. lepraespecific cell-mediated immunity (CMI) are seen in multibacillary $(\mathrm{MB})$ patients while paucibacillary $(\mathrm{PB})$ leprosy has strong M. leprae-specific CMI and low or undetectable antibody levels (2). Over the years, several leprosy serologic tests using different methodologies and antigens have been reported. Lateral flow, dipstick, particle agglutination, and enzyme-linked immunosorbent assays (ELISAs) mostly employing the $M$. leprae-specific native or synthetic di- or trisaccharide epitope of the phenollic glycolipid-I antigen (PGL-I) chemically linked to bovine or human serum albumin via octyl or phenyl group (ND-O or NT-P) have been tested in field-based studies (3-9). These studies have shown high IgM positivity in $\mathrm{MB}$ patients and low positivity in $\mathrm{PB}$ patients $(5,10,11)$. After the completion of Mycobacterium tuberculosis and $M$. leprae genomes $(12,13)$, new $M$. leprae-specific proteins have been screened for serology and CMI-tests. The ML0405 and the ML2331 proteins were shown to be highly recognized by $\mathrm{MB}$ patients and were later engineered as the di-fusion LID-1 antigen (Leprosy Infectious Disease Research Institute/IDRI Diagnostic-1) (14-16). Positivity to IgG ELISAs to LID-1 is also proportional to the patient's bacillary load $(14,15,17,18)$. More recently, ND-O-LID antigen, a single fusion complex of natural disaccharide-octyl epitope (ND-O) of PGL-I and LID-1 has been used for the simultaneous, detection of IgM and IgG antibodies in lateral flow test and ELISA (19-23).

Leprosy is a treatable and curable disease and for multidrug therapy (MDT) implementation, patients are classified either as $\mathrm{MB}$ (six or more skin lesions, LL, BL, BB forms) or PB (up to five skin lesions, TT and BT forms) (24), The standard leprosy MDT treatment comprises two different regimens: 12 months with rifampicin, dapsone, and clofazimine for $\mathrm{MB}$ patients and 6 months with rifampicin and dapsone for PB patients (24). In $\mathrm{MB}$ patients, MDT reduces $M$. leprae-specific antibody titers suggesting the application of serology to monitor treatment efficacy (25-34). In 2007, an open-label, randomized clinical trial was conducted to compare the main outcomes [relapses, leprosy reactions, bacilloscopic index (BI) decline] of patients treated with the regular WHO MDT (R-MDT) and a 6-month uniform MDT regimen (U-MDT, rifampicin, dapsone, and clofazimine) for both $\mathrm{PB}$ and $\mathrm{MB}$ leprosy, regardless of any classification [Clinical Trial for Uniform Multidrug Therapy Regimen for Leprosy Patients in Brazil (U-MDT/CT-BR)] (35-42). As part of the U-MDT/CT-BR, a bank comprising sequential serum samples collected before, during and after R-MDT and U-MDT was assembled. This study, reports the impact of the U-MDT and the R-MDT on leprosy serology to PGL-I, LID-1, and ND-O-LID antigens and the kinetics of antibody responses at different time points in both treatment groups.

\section{MATERIALS AND METHODS}

\section{Study Population}

Our study group comprises only $\mathrm{MB}$ patients from $U-M D T /$ $C T-B R$ (Dona Libânia, Fortaleza, Ceará state and Alfredo da Matta, Manaus, Amazonas state), recruited from 2007 to 2015 that had positive bacilloscopy and at least three serum samples collected during monitoring (36). Serum samples tested were collected before MDT (M0/month zero), from 1 to 12 months after the start of MDT (M1-M12) and at the first and second year after the conclusion of treatment (R-MDT first and second year: 24 and 36 months after treatment conclusion, respectively and U-MDT first and second year: 18 and 30 months after treatment conclusion, respectively). Details of patients' recruitment, diagnosis, and main follow-up outcomes have been previously described (35-43).

\section{Leprosy Serology}

Serologic reactivity to $M$. leprae antigens was assessed by ELISA using the following antigens: natural trisaccharide-phenyl-BSA (NT-P-BSA) a semi-synthetic analog of PGL-I (batch: Nara XVI61; Dr. Fujiwara, Japan), Leprosy Infectious Disease Research Institute Diagnostic-1 (LID-1) (batch: ago 2012, IDRI, USA) and the single fusion complex (ND-O-LID-batch: 17 August 2012, IDRI, USA).

\section{Detection of IgM Antibodies to PGL-I}

Serum IgM antibodies to PGL-I were detected by ELISA as previously described (3). PolySorp 96-well plates (Nunc, Roskilde, Denmark) were coated with $50 \mu \mathrm{l} /$ well of $0.01 \mathrm{mg} / \mathrm{mL}$ of the sugar 
component of NT-P-BSA or BSA and blocked with 1\% BSA/PBS. Serum samples diluted $1 / 200$ in PBS-Tween containing $10 \%$ normal goat serum/NGS (Sigma-Aldrich, St. Louis, MO, USA) were tested in NT-P-BSA and in BSA coated wells. After incubation and washings, horseradish peroxidase/HRP-conjugated anti-human IgM (Immuno Chemicals, St. Louis, MO, USA) was added. In order to control plate-to-plate and day-to-day variation, a positive reference serum was added in duplicate on each plate. After incubation and washings, peroxidase color substrate (TMB, Sigma-Aldrich, St. Louis, MO, USA, Homemade) was added and the reaction was quenched by the addition of $2.5 \mathrm{~N}$ $\mathrm{H}_{2} \mathrm{SO}_{4}$, when the $\mathrm{OD}$ at $450 \mathrm{~nm}$ from reference serum reached an $\mathrm{OD}$ value of 0.6 . The $\mathrm{OD}$ was measured at $450 \mathrm{~nm}$ using a Bio-Rad micro plate reader (Life Science, Hercules, CA, USA). The final OD was calculated by subtracting the OD of BSA coated wells from OD values of NT-P-BSA coated wells. The cutoff was defined as $\mathrm{OD}>0.25$ as previously described (5).

\section{Detection of IgG Antibodies to LID-1 and Detection of IgM and IgG Antibodies to ND-O-LID}

Serum IgG antibodies to LID-1 were detected by ELISA. Polysorp 96-well plates (Corning Costar, NY, USA) were coated with $100 \mu \mathrm{l} /$ well of $1 \mu \mathrm{g} / \mathrm{mL} \mathrm{LID-1}$ or with $100 \mu \mathrm{l} /$ well of $0.25 \mu \mathrm{g} / \mathrm{mL}$ ND-O-LID. Blocking was performed with PBS-T 1\% BSA. Serum samples diluted $1 / 200$ in PBS-T-10\% NGS were added in duplicate and incubated for $2 \mathrm{~h}$ at room temperature. Plates were washed and incubated for $1 \mathrm{~h}$ with HRP-conjugated anti-human IgG (Southern Biotech, Birmingham, AL, USA) for anti-LID-1 serology or for anti-ND-O-LID serology plates were incubated with anti-human IgG (Southerm Biotech, Birmingham, AL, USA) plus anti-human IgM (Immuno Chemicals, St. Louis, MO, USA). After washings, reactions were developed with peroxidase color substrate (KPL, Gaithersburg, MD, USA) and quenched by the addition of $1 \mathrm{~N} \mathrm{H}_{2} \mathrm{SO}_{4}$. The optical density (OD) was determined (Bio-Rad microplate reader, Life Science, Hercules, CA, USA) at $450 \mathrm{~nm}$. For anti-LID-1 serology, the cutoff was calculated as two times the SD of the OD of sera from healthy endemic controls, such that samples with $\mathrm{OD}>0.3$ were considered positive (15). As previously described, the anti-ND-O-LID serology threshold for positive responses was considered OD $>0.923$ (20). The results of serologic tests were expressed as the mean OD of duplicates.

\section{Statistical Analyses}

Antibody levels were measured taking into account the medians of the OD at different time points in each treatment group. The percentage of positive samples was calculated based on the number of samples with OD above the cutoff established for each test at each time point. The statistical analyses performed in this study aimed mainly to answer if the data supported the hypothesis that the serological results have a time trend after the beginning of treatment, reflecting the reduction in bacillary load, and if this trend differed between the two treatment groups. The first statistical analysis employed Kruskal-Wallis test one-way analysis of variance for comparison of multiple groups and the Mann-Whitney $U$-test for comparison between two groups comparing data of all patients at different time points. Results were considered statistically significant when $p$ values $<0.05$ were obtained. The intraindividual decay of serology among patients from R-MDT and U-MDT was evaluated employing mixed effects hierarchical/multilevel regression analyses using STATA software $(44,45)$. The multilevel analyses considered the individual serological results to each different antigen during different time points of follow-up. For these analyses, the independent variable was the serological result, the dependent variables were time and treatment group, and the group variable was patient ID. These analyses allowed the investigation of the effects that vary by group (each patient) and estimate group level averages in which each patient has his own time trend where one measure is not independent of the previous one.

\section{RESULTS}

\section{Main Characteristics of Study Population}

In this study, we have assessed the serologic reactivity of 3,400 sequential serum samples collected at different time points, from $263 \mathrm{MB}$ leprosy patients, with positive bacilloscopy, enrolled at U-MDT/CT-BR and treated either with the R-MDT or U-MDT regimens. Among $263 \mathrm{MB}$ patients, 56 were from Amazonas State and 207 came from Ceará State. In our study group, 54\% (142 out of 263) received U-MDT and 46\% (121 out of 263) were treated with R-MDT. For each patient, a median of 13 sequential serum samples (range: 3-21 samples) was collected at different time points: before MDT (M0), during treatment (M1-M6 for U-MDT and M1-M12 for R-MDT) and after treatment conclusion (first and second year). The main clinical and laboratory features of MB leprosy patients included in this serological study were similar (Table 1). The majority of MB leprosy patients was male, and patients from R-MDT and U-MDT groups had similar age (median age: 41 and 40.5 years, respectively). The majority of patients from the R-MDT and U-MDT groups was classified as BL and LL leprosy (R-MDT: 88\%, 107 out of 121; U-MDT: 90\%, 128 out of 142). The median of the BI in the R-MDT group was 3.6 (0.2-5.75 range) and 3.8 (0.2-6 range) in the U-MDT group. In the R-MDT group, $61 \%$ (69 out of 113 ) developed a reactional episode, $67 \%$ had reversal reaction (RR) (46 out of 69) of these, $11 \%$ (5 out of 46 ) at diagnosis and $89 \%$ during follow-up (41 out of 46). In the R-MDT, 33\% (23 out of 69) had erythema nodosum leprosum/ENL, of these $4 \%$ ( 1 out of 23 ) at diagnosis and $96 \%$ (22 out of 23) during follow-up. In the U-MDT group, $62 \%$ was reactional (82 out of 132) of these $72 \%$ had RR (59 out of 82 ) of these, $14 \%$ ( 8 out of 59 ) at diagnosis and $86 \%$ during follow-up (51 out of 59). In the U-MDT, $28 \%$ (23 out of 82 ) had ENL, of these $9 \%$ (2 out of 23) at diagnosis and 91\% (21 out of 23) during follow-up.

\section{Decline of Anti-PGL-I, Anti-LID-1, and Anti-ND-O-LID M. leprae-Specific Antibody Levels in the U-MDT and R-MDT Groups During Follow-Up}

Compared to baseline results, in both R-MDT and U-MDT groups, a significant decline in anti-PGL-I levels was observed 
upon treatment (Figure 1A). At baseline, the median OD in the R-MDT group was 0.437 and in the U-MDT group, it was 0.516 ; after 5 months of treatment, the median OD was 0.325 in the

TABLE 1 | Main clinical and laboratory characteristics of the 263 MB leprosy patients enrolled at U-MDT/CT-BR stratified according to the treatment group.

\begin{tabular}{lcc}
\hline & R-MDT $(\boldsymbol{n}=\mathbf{1 2 1})$ & U-MDT $(\boldsymbol{n}=\mathbf{1 4 2})$ \\
\hline $\begin{array}{l}\text { Gender (male/female) } \\
\text { Age (years) median }\end{array}$ & $86 / 35$ & $105 / 37$ \\
(range) & $41(8-65)$ & $40.5(7-65)$ \\
R\&J classification & $12 \mathrm{BT}, 2 \mathrm{BB}, 75 \mathrm{BL}, 32 \mathrm{LL}$ & $9 \mathrm{BT} ; 5 \mathrm{BB} ; 89 \mathrm{BL}, 39 \mathrm{LL}$ \\
Bl median (range) & $3.6(0.2-5.75)$ & $3.8(0.2-6)$ \\
$\begin{array}{l}\text { Development of } \\
\text { reactions }\end{array}$ & $73 / 121(60 \%)$ & $88 / 142(62 \%)$ \\
Type and moment & RR: $50 / 73$ & RR: $62 / 88$ \\
of development of & At diagnosis: $7 / 50$ & At diagnosis: $8 / 62$ \\
reactions & During follow-up: $43 / 50$ & During follow-up: $54 / 62$ \\
& ENL: $23 / 73$ & ENL: $26 / 88$ \\
& At diagnosis: $1 / 23$ & At diagnosis: $2 / 26$ \\
& During follow-up: $22 / 23$ & During follow-up: $24 / 26$
\end{tabular}

R\&J, Ridley and Jopling classification system; BB, borderline borderline; BL, borderline lepromatous; LL, lepromatous leprosy; BI, bacilloscopic index; R-MDT, regular multidrug therapy; U-MDT, uniform multidrug therapy; RR, reversal reaction; ENL, erythema nodosum leprosum; MDT, multidrug therapy; BT, borderline tuberculoid.
R-MDT group and 0.424 in the U-MDT group $(p=0.035$ and $p=0.04$, respectively). In the R-MDT group considering baseline (M0) serology, there was a significant decline of the anti-PGL-I levels in the subsequent months (M0 vs M5, $p=0.03$; M0 vs M7, $p=0.09 ; \mathrm{M} 0$ vs M8, $p=0.01 ; \mathrm{M} 0$ vs M9, $p=0.01 ; \mathrm{M} 0$ vs M10, $p=0.02 ; \mathrm{M} 0$ vs M11, $p=0.007$; M0 vs M12, $p=0.001, \mathrm{M} 0$ vs first year, $p=0.02$; M0 vs second year, $p<0.0001$ ) (Figure 1A). In the U-MDT group, anti-PGL-I levels at diagnosis also reduced during and after treatment (M0 vs M5, $p=0.04 ; \mathrm{M} 0$ vs M6, $p=0.02 ; \mathrm{M} 0$ vs M7, $p=0.01 ; \mathrm{M} 0$ vs M8, $p=0.001 ; \mathrm{M} 0$ vs M9, $p=0.003 ; \mathrm{M} 0$ vs M10, $p=0.0007 ; \mathrm{M} 0$ vs M11, $p=0.001 ; \mathrm{M} 0$ vs M12, $p<0.0001$, M0 vs first year, $p=0.03$; M0 vs second year, $p=0.0004)$ (Figure 1A).

Regarding serological results in the R-MDT group, a significant decline in IgG anti-LID-1 antibodies was observed comparing baseline and M6 (median ODs at M0 = 1.386 vs M6 = 1.068; $p=0.024$ ) (Figure 1B). In the R-MDT group, anti-LID-1 antibodies continued to decrease during subsequent months (M0 vs M7, $p=0.0003$; M0 vs M8, $p<0.0001$; M0 vs M9, $p=0.0009$; M0 vs $\mathrm{M} 10, p<0.0001 ; \mathrm{M} 0$ vs M11, $p=0.0005 ; \mathrm{M} 0$ vs M12, $p<0.0001)$, and at the first and second year posttreatment (M0 vs first year, $p=0.002$; M0 vs second year, $p<0.0001$ ) (Figure 1B). In the U-MDT group, anti-LID-1 antibodies declined significantly
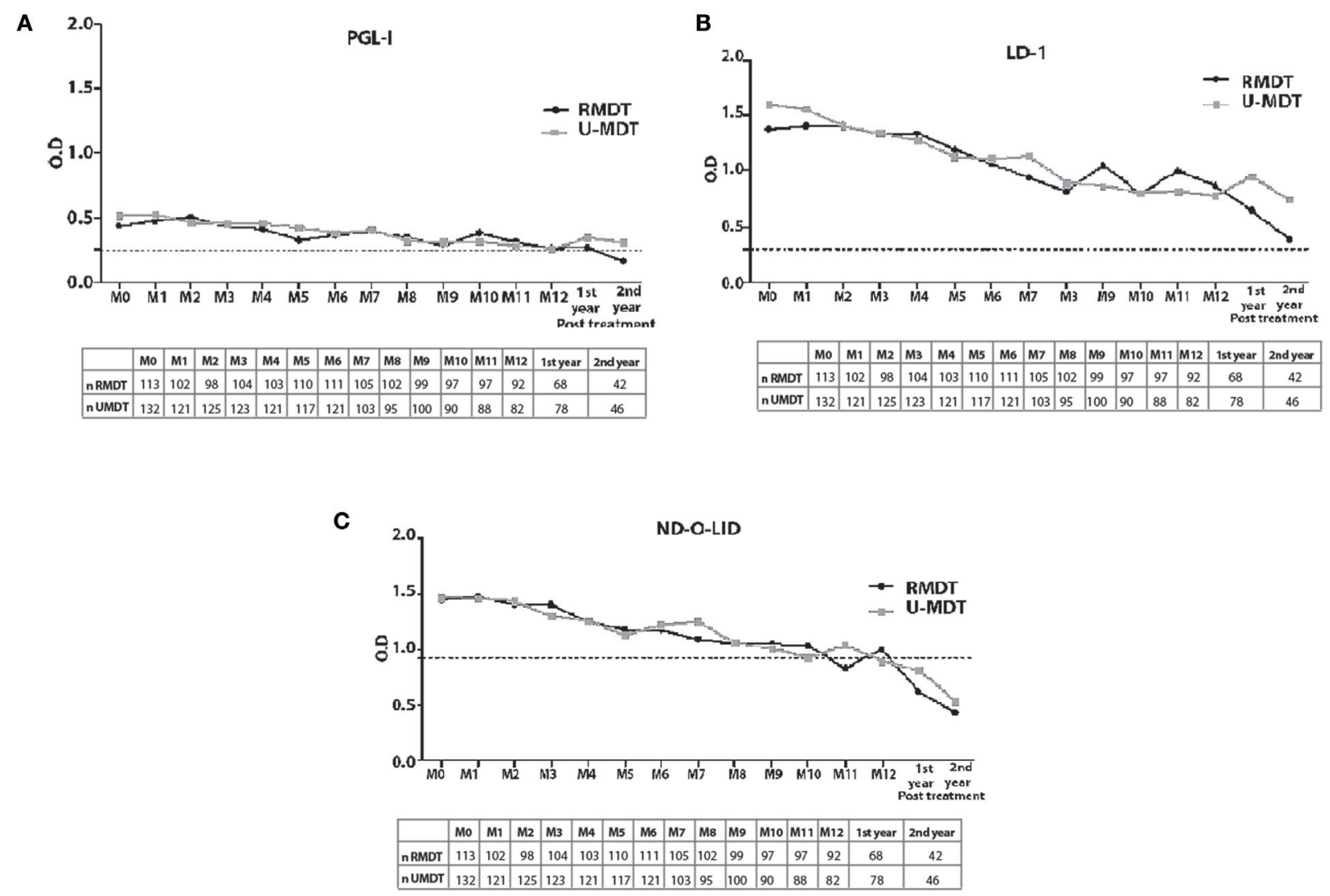

FIGURE 1 | Kinetic of Mycobacterium leprae-specific antibody responses in multibacillary treated with regular multidrug therapy (R-MDT) (closed black circles) and uniform multidrug therapy (U-MDT) (gray closed squares) from baseline month zero (M0) to month 12 (M12) after starting treatment, and after the first and second year of treatment conclusion. (A) Anti-phenollic glycolipid-I antigen serology; (B) anti-LID-1 serology; (C) anti-ND-O-LID serology. Each point represents the median optical density (OD) value of each group. The dotted horizontal lines indicate cutoff points of each serological test. 
from baseline to the fourth month of treatment (median ODs at $\mathrm{M} 0=1.605$, at $\mathrm{M} 4=1.279 ; p=p=0.003$ ). Compared to baseline data/M0, anti-LID-1 antibodies decreased after M5 (M0 vs M5, $p<0.0001$; M0 vs M6, $p<0.0001 ;$ M0 vs M7, $p=0.0003$ and M0 vs M8, M0 vs M9, M0 vs M10, M0 vs M11 and M0 vs M12, $p<0.0001$ ) and at the first and second year posttreatment (M0 vs first year and M0 vs second year, $p<0.0001$ ) (Figure 1B).

In the R-MDT group, serology using ND-O-LID antigen showed a significant decline in antibody levels from baseline (M0) to the seventh month of treatment (M7) (median ODs at $\mathrm{M} 0=1.449$ vs $\mathrm{M} 7=1.092 ; p=0.005)$ (Figure 1C). Among patients treated with R-MDT, anti-ND-O-LID antibodies continued to decrease after M8 (M0 vs M8, $p=0.003$; M0 vs M9, $p=0.002 ; \mathrm{M} 0$ vs M10, $p=0.0007$; M0 vs M11, $p=0.0006$; M0 vs M12, $p<0.0001$, M0 vs first year and M0 vs second year posttreatment, $p<0.0001$ ) (Figure 1C). In the U-MDT group, a significant decline in anti-ND-O-LID antibodies was observed from baseline to the fifth month (median ODs M0 $=1.466$ vs M5 $=1.126 ; p=0.006)$ and antibody levels decreased after M6 (M0 vs M6, $p=0.02 ; \mathrm{M} 0$ vs M7, $p=0.01 ; \mathrm{M} 0$ vs M8, $p=0.0006$; M0 vs M9, $p=0.0003$; M0 vs M10, $p=0.0001$; M0 vs M11, $p=0.0006$ and M0 vs M12, $p<0.0001)$ and in the first year and second year posttreatment $(p<0.0001)$ (Figure 1C).

\section{Decline in the Positivity Rates for Anti- PGL-I, LID-1, and ND-O-LID Antibodies Among U-MDT and R-MDT Groups}

At baseline, $71 \%$ of $\mathrm{MB}$ patients who received R-MDT was antiPGL-I positive, after 6 months MDT (M6) positivity declined to $63 \%(p>0.05)$, and at the end of treatment (M12) $46 \%$ of patients remained anti-PGL positive (M0 vs M12, $p=0.0001$ ) (Figure 2A). In the first-year posttreatment, 43.5\% (27 out of 62) was positive and in the second-year posttreatment, 38\% (15 out of 39) remained positive. Regarding baseline serology, the decline in anti-PGL-I positivity in the R-MDT group was statistically significant (M0 vs M12, M0 vs first year, M0 vs second year posttreatment, $p=0.0001$ ).

In the U-MDT group, 74\% was anti-PGL-I positive at baseline, $66 \%$ at 6 months (M0 vs M6, $p>0.05$ ) and at M12, 51\% remained anti-PGL-I positive (M0 vs M12, $p=0.0002$ ) (Figure 2B). Positivity rate in the first-year posttreatment was 58\% (43 out of 74) and in the second-year posttreatment $44 \%$ (18 out of 41 ) remained positive. Compared to serology at diagnosis, in the U-MDT group, the reduction in anti-PGL-I positivity rate was statistically significant ( $\mathrm{M} 0$ vs first year, $p=0.008$; M0 vs second year posttreatment, $p=0.0001)$. Anti-PGL-I positivity rates in patients from the R-MDT and the U-MDT regimens were similar at different time points: M0, M6, M12, and second year posttreatment $(p>0.05)$ (Figures 2A,B). In the first-year posttreatment, anti-PGL-I positivity rate was higher in the U-MDT than in the R-MDT group ( $p=0.04)$.

In the R-MDT group, anti-LID-1 positivity rate at baseline was $88 \%$, after 6 months of treatment (M6) 84\% remained positive and at the end of MDT (M12), 79\% had antibodies above the positivity threshold (Figure 2C). In the first year posttreatment, 62\% (42 out of 68 ) of patients was seropositive and in second year $61 \%$ (26 out of 42) remained positive. In the R-MDT group, the percentage of anti-LID-1 positivity was similar at M0, M6, and M12 ( $p>0.05)$. However, a statistically significant reduction in positivity rate was seen comparing M0 and first year $(p=0.0002)$ and M0 and second year posttreatment $(p=0.0001)$. In the U-MDT group, before treatment $88 \%$ of the patients was anti-LID-1 positive, after 6 months/end of treatment $86 \%$ was positive and at M12 $80 \%$ remained positive (Figure $2 \mathrm{D}$ ). In first year posttreatment, $78 \%$ (61 out of 78 ) was anti-LID-1 positive and in second year positivity was $65 \%$ (30 out of 46 ). The positivity rate to LID-1 antigen was similar at different time points: M0, M6, and M12 $(p>0.05)$. The reduction in the positivity rate to LID-1 serology was significant comparing M0 vs first year and M0 vs second year posttreatment ( $p=0.03$ and 0.0002 , respectively). Positivity rates to LID-1 serology between U-MDT and R-MDT groups were similar at M0, M6, and M12 ( $p>0.05$ ) (Figures 2C,D). In the first year posttreatment, a statistically significant difference was observed between U-MDT and R-MDT regimens $(p=0.01)$.

Anti-ND-O-LID positivity in the R-MDT group was $73 \%$ at baseline, after 6 months MDT (M6) positivity was $63 \%(p>0.05)$ and at the end of treatment (M12), 54\% of patients remained positive (M0 vs M12 $p=0.003$ ) (Figure $2 \mathrm{E}$ ). At the first year posttreatment, $31 \%(21 / 68)$ of patients remained positive and in the second year $17 \%$ were seropositive ( 7 out of 42 ) (M0 vs first year, $p=0.0001, \mathrm{M} 0$ vs second year, $p=0.0001)$. In U-MDT group, before treatment, $73 \%$ of the patients was anti-ND-O-LID positive, after 6 months/end of treatment, positivity was $62 \%$ and at M12, 47\% remained positive (Figure 2F). In the U-MDT group, the decrease in anti-ND-O-LID positivity rate during the first 12 months of monitoring was statistically significant (M0 vs $\mathrm{M} 12, p=0.0006)$. There was a significant decline in the positivity rate to anti-ND-O-LID serology from baseline to the first year posttreatment and from baseline to the second year after treatment conclusion ( $\mathrm{M} 0$ vs first year, $p=0.0001 ; \mathrm{M} 0$ vs second year, $p=0.0001)$. Anti-ND-O-LID positivity rates between U-MDT and R-MDT were similar at M0, M6, and M12 ( $p>0.05)$ (Figures 2E,F). In the first year after treatment conclusion, a statistically significant difference in positivity rate was observed between U-MDT and R-MDT regimens ( $p=0.03$ ).

Our study group of $263 \mathrm{MB}$ patients included the BT, BB, BL, and LL categories, according to the adapted Ridley and Jopling classification system used. Among $263 \mathrm{MB}$ patients, 28 were either BT $(n=14)$ or BB $(n=14)$, representing $12 \%$ of the R-MDT group and $10 \%$ of the U-MDT group. BT and BB have lower $\mathrm{BI}$ compared to $\mathrm{BL}$ and LL categories and as serology reflects the $\mathrm{BI}$ of the patient, the impact of BT/BB patients on the serology was analyzed by removing these 28 patients from the groups and by comparisons of results of the groups with and without BT/BB. Our results showed that difference in serological results obtained upon exclusion of BT and BB patients was statistically significant only for LID-1 serology at M6 while for all other antigens and time points there was no statistically significant change in the positivity rate by comparing the whole group of $\mathrm{BT}, \mathrm{BB}, \mathrm{BL}, \mathrm{LL}$, and the group of BL and LL patients (Table S1 in Supplementary Material).

The association between the $\mathrm{BI}$ at diagnosis and the serologic responses to the three antigens was evaluated 12 months after 

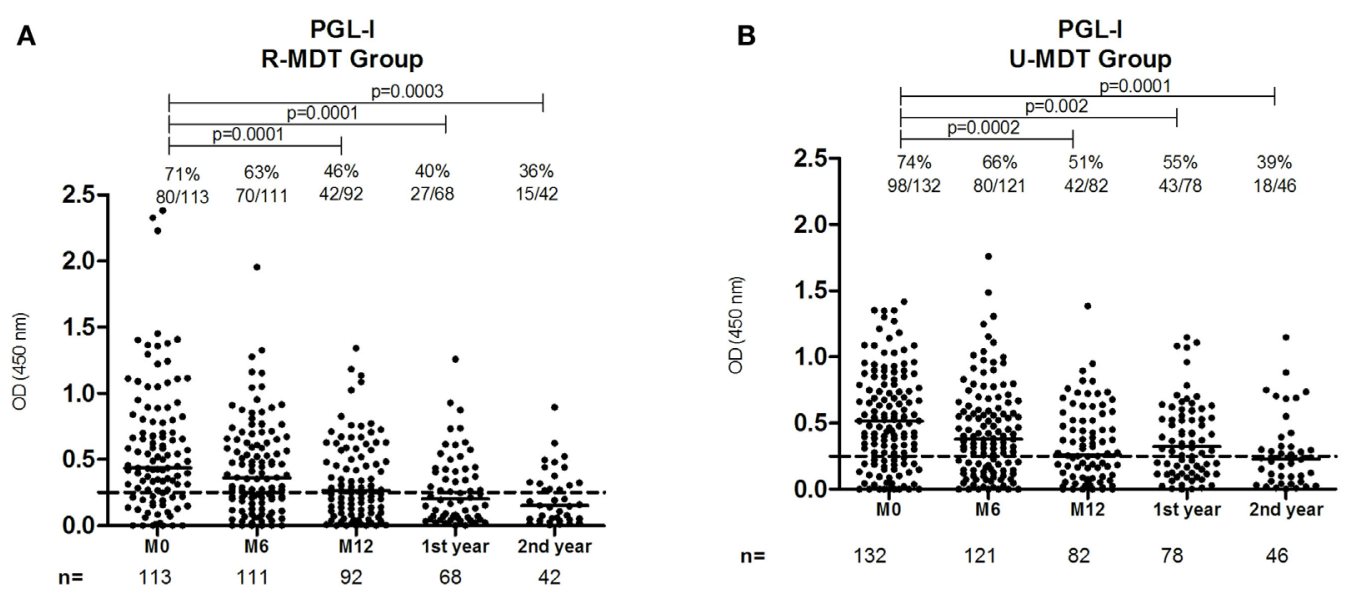

C

LID-1

R-MDT Group

D

LID-1
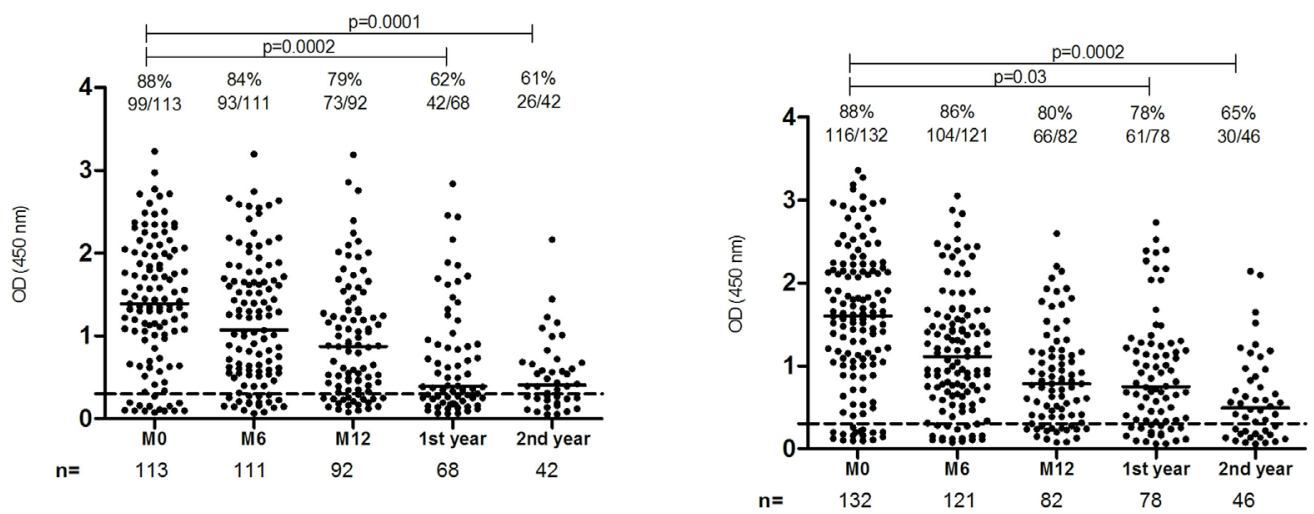

E

ND-O-LID R-MDT Group

F

ND-O-LID
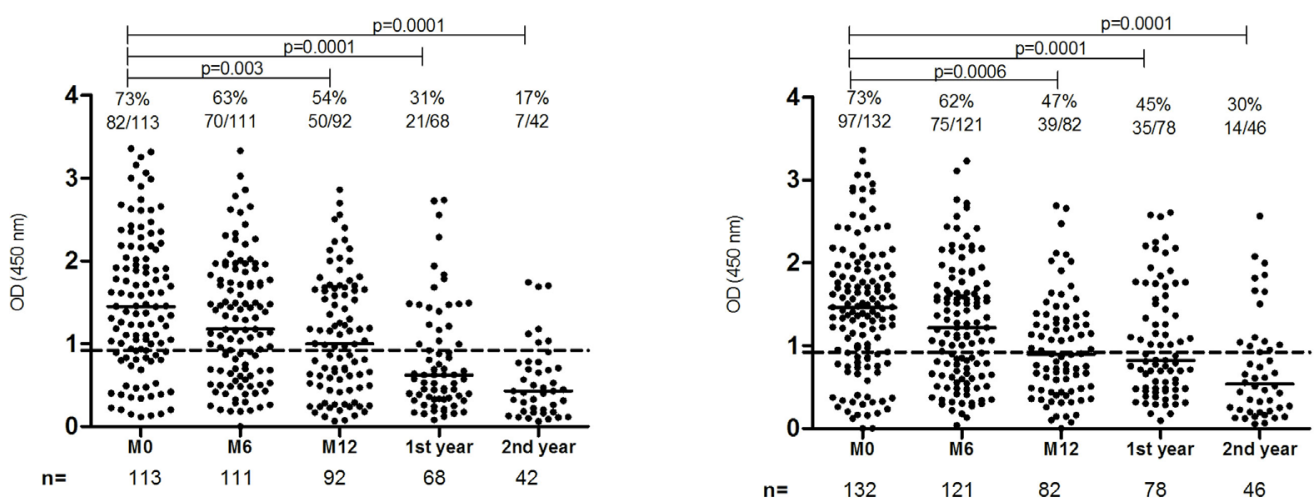

FIGURE 2 | Serologic reactivity to phenollic glycolipid-I antigen (PGL-I), LID-1, and ND-O-LID antigens among multibacillary patients from the regular multidrug therapy (R-MDT) and uniform multidrug therapy (U-MDT) groups at different time points MO, M6, M12, and first and second year posttreatment: anti-PGL-I positivity rates in (A) R-MDT group, (B) U-MDT group; anti-LID-1 positivity rates in (C) R-MDT group, (D) U-MDT group; anti-ND-O-LID positivity rates in (E) R-MDT group, (F) U-MDT group. Each point represents the mean optical density (OD) of duplicates of each individual patient. The median OD value of each group is represented by the horizontal line and the traced line represents the different cutoffs (PGL-I OD > 0.25, LID-1 OD > 0.3, and ND-O-LID OD > 0.923). The number above each dataset is the percent of positive responses. The $p$ value refers to differences in positivity rates at different time points.

initiation of both regimens (Figures 3A,B). In the R-MDT group, these analyses included 32 patients with $\mathrm{BI} \leq 3$ (median $=2$, range $0.2-3.0)$ and 56 patients with $\mathrm{BI}>3$ (median $=4$, range
3.2-5.75). For all antigens tested, the median of OD at M12 was higher among patients with initial $\mathrm{BI}>3$ compared to patients with $\mathrm{BI} \leq 3(p<0.05)$ especially for LID-1 serology $(p=0.0002)$ 
(Figure 3A). In the U-MDT group, 28 patients with $\mathrm{BI} \leq 3$ (median $\mathrm{BI}=1.775$, range $0.2-3$ ) and 51 patients with $\mathrm{BI}>3$ (median $\mathrm{BI}=4$, range 3.2-6) had their serologic responses compared at M12. In this group, a higher OD value to all three antigens was seen in patients with $\mathrm{BI}>3$, however, reaching statistical significance only for LID-1 antigen ( $p=0.009)$ (Figure 3B).

\section{Intraindividual Decline of Anti-PGL-I, Anti-LID-1, and Anti-ND-O-LID M. leprae- Specific Antibody Levels in the U-MDT and R-MDT Groups}

Multilevel regression analyses were performed with serologic results to PGL-I (Figure 4A), LID-1 (Figure 4B), and ND-O-LID antigens (Figure 4C) at different time points of follow-up in 850 samples of 244 patients from both R-MDT and U-MDT groups. These analyses showed that the difference in serologic decay to all three antigens was dependent upon time only. Similar decay of serology to all three antigens was seen among patients from both R-MDT and U-MDT (Figures 4A-C; Table 2).

\section{DISCUSSION}

This study including cross sectional and intraindividual analyses showed that both shorter 6 months U-MDT and standard 12 months R-MDT using rifampicin, dapsone, and clofazimine had a similar effect on leprosy specific serology, reducing the antibodies of $\mathrm{MB}$ leprosy patients to three well-characterized $M$. leprae antigens: PGL-I, LID-1, and anti-ND-O-LID. Serologic responses detected at baseline declined during the course of therapy and continued to decline after discontinuation of specific treatment. Multilevel analyses of intraindividual responses showed that for both treatment regimens R-MDT and U-MDT, the decay of serologic reactivity to all three antigens tested was dependent on time only. In leprosy, serology is considered a surrogate marker of the bacillary load and a previous study has shown that MB patients from the R-MDT and the U-MDT groups had similar reduction in the bacillary load (39). In both treatment groups, despite minor oscillations, the pattern of decline in antibody levels was similar for all three $M$. leprae antigens tested. Our results indicate that, regardless of the duration of the treatment regimens for multibacillary patients, antibodies decline overtime during and after treatment interruption. This is the first description of the dynamics of antibody responses to three $M$. leprae-specific antigens among a well-characterized cohort of MB patients, mostly with high bacillary load at diagnosis, who was treated with two different MDT regimens and rigorously monitored during a clinical trial in Brazil. Our study sample contained a robust collection of 3,400 serum samples, including around 13 sequential samples per patient, which were collected since diagnosis over a 3-year period and this serologic study revealed the kinetic of specific antibody responses during this period.

The decrease in antibody levels following MDT, especially to PGL-I, has been well reported in previous studies showing longitudinal data $(25-32,34)$. In the U-MDT group, antibody titers to all three antigens tested, decreased during the 6-month treatment and despite some variation, anti-PGL-I antibodies continued to decline after treatment discontinuation until the first and second year after MDT conclusion. However, among MB patients most of them with high bacillary load at diagnosis, despite the decline in antibody levels, most patients remained seropositive 2 years after treatment conclusion. The decline in anti-PGL-I positivity from baseline to 2 years after treatment discontinuation was similar in both treatment groups when $38 \%$ in the R-MDT and $44 \%$ in the U-MDT showed serological responses above the cutoff for
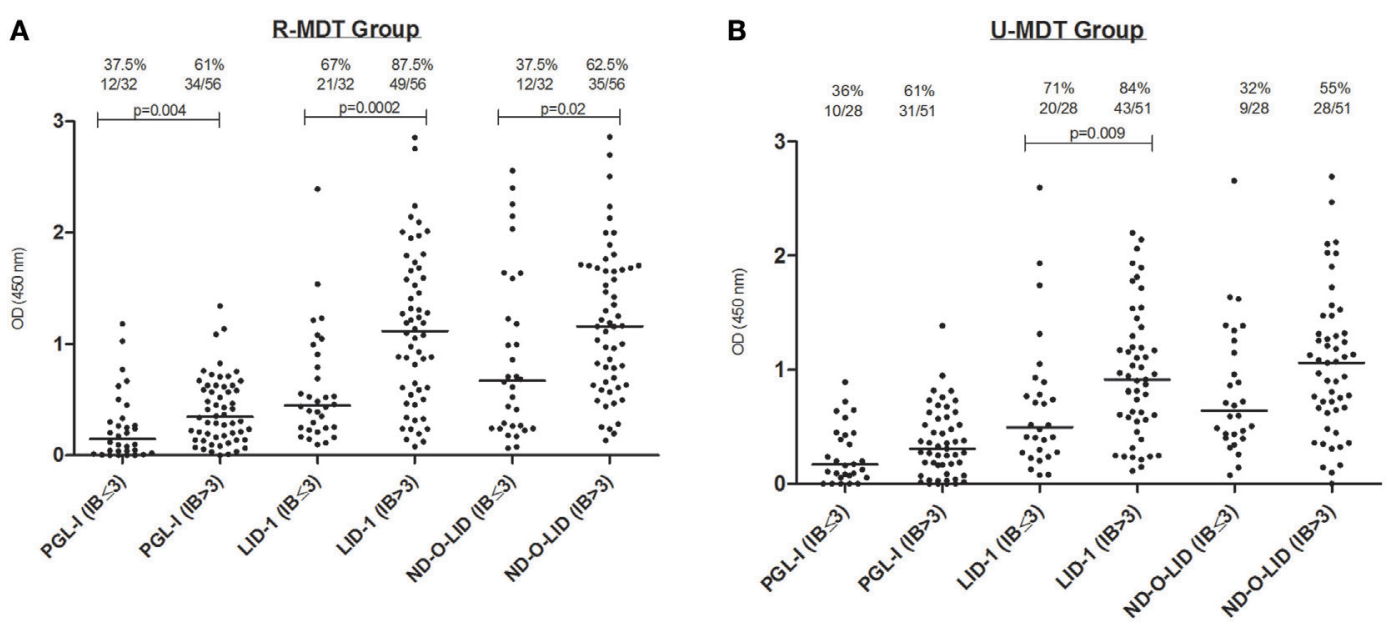

FIGURE 3 | Serologic reactivity at 12 months to phenollic glycolipid-I antigen (PGL-I), LID-1, and ND-O-LID antigens among multibacillary (MB) patients stratified by $\mathrm{BI} \leq 3$ and $\mathrm{BI}>3$ from the regular multidrug therapy (R-MDT) (A) and uniform multidrug therapy (U-MDT) (B) groups. Each point represents the mean OD of duplicates of each individual patient. The median OD value of each group is represented by the horizontal line and the traced line represents the different cutoffs (PGL-I OD > 0.25, LID-1 OD > 0.3, and ND-O-LID OD > 0.923). The number above each dataset is the percent of positive responses. The $p$ value refers to differences in medians at different time points. OD, optical density; BI, bacilloscopic index. 


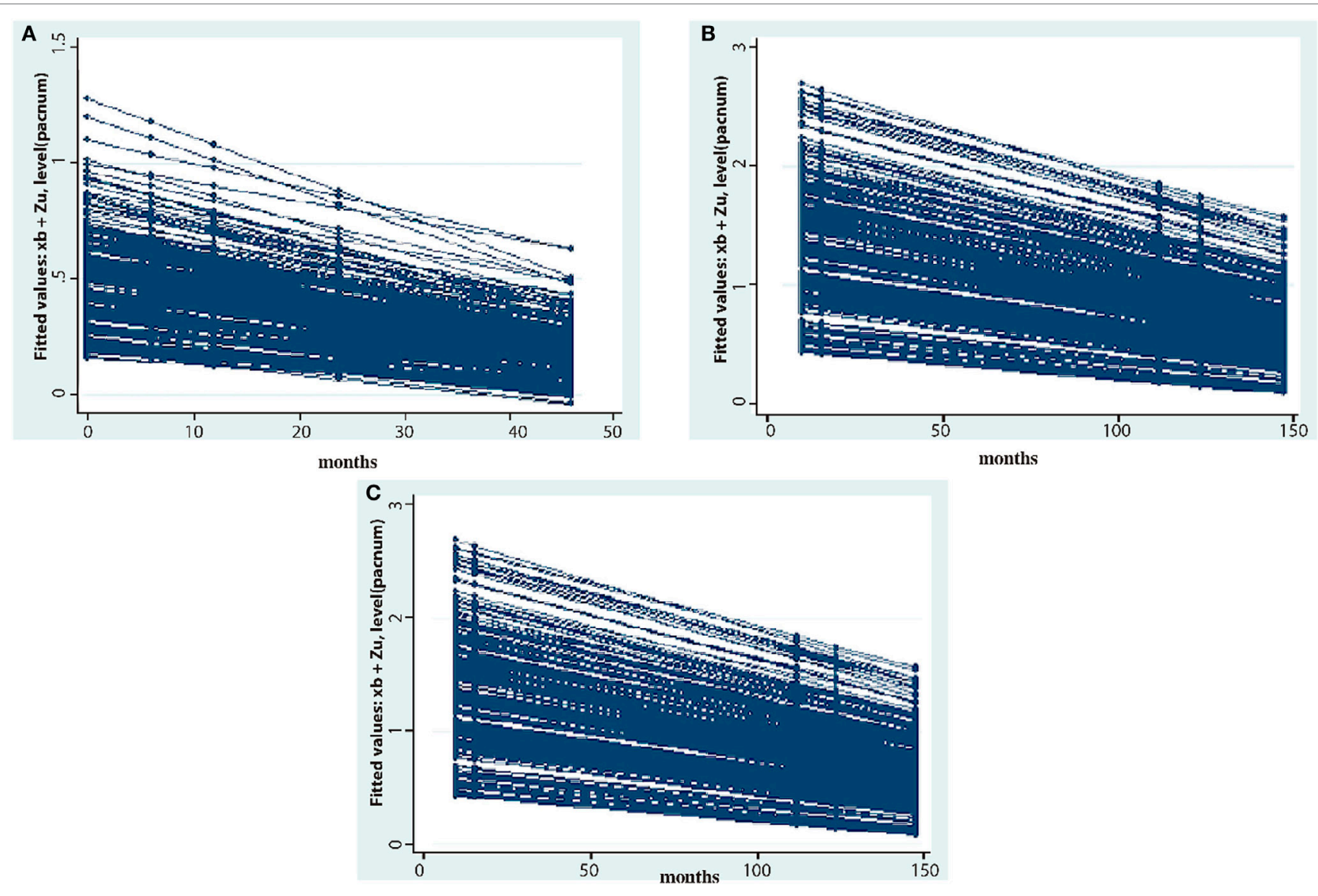

FIGURE 4 | Mixed effect multilevel analyses of 244 patients treated either with regular multidrug therapy (R-MDT) or the uniform multidrug therapy (U-MDT) showing the decay of antibody responses to different Mycobacterium leprae antigens (optical density, $y$ axis) overtime (months, $x$ axis): (A) phenollic glycolipid-I antigen, (B) LID-1, (C) ND-O-LID.

TABLE 2 | Results of adjusted mixed multilevel regression analyses of anti-PGL-I, anti-LID-1, and anti-NDO-LID serology overtime in leprosy patients treated with R-MDT and U-MDT.

\begin{tabular}{lcccccc}
\hline \multicolumn{1}{c}{ Coefficient } & SE & $\boldsymbol{z}$ & $\boldsymbol{p}>\boldsymbol{z}$ & $\mathbf{9 5 \%}$ Confidence interval \\
\hline Anti-PGL-I & & & & & \\
Month - & & & & & & \\
Group & -0.006871 & 0.0007285 & -9.43 & 0 & -0.0082988 & -0.0054431 \\
_Cons & 0.5085021 & 0.0333417 & -0.11 & 0.916 & -0.0688648 & 0.0618322 \\
Anti-LID-1 & 0.1160942 & 4.38 & 0 & 0.2809617 & 0.7360426 \\
Month -0.0051023 & 0.0003361 & -15.18 & 0 & -0.005761 & -0.0044436 \\
Group -0.0571696 & 0.0719866 & -0.79 & 0.427 & -0.1982608 & 0.0839216 \\
_Cons & 1.618 .408 & 0.2525812 & 6.41 & 0 & 1.123 .358 & 2.113 .458 \\
Anti-NDO-LID & & & & & \\
Month -0.0164264 & 0.0012887 & -12.75 & 0 & -0.0189521 & -0.0139006 \\
Group & 0.0016501 & 0.074866 & 0.02 & 0.982 & -0.1450847 & 0.1483848 \\
Cons & 1.379 .876 & 0.2617582 & 5.27 & 0 & 0.866839 & 1.892 .912 \\
\hline
\end{tabular}

The mixed effects multilevel regression analyses evaluated the individual results of anti-PGL-I, anti-LID-1, and anti-ND-O-LID serology during follow-up considering the serological result as the independent variable (constant), the dependent variables were time (month) and treatment group (R-MDT and U-MDT) and the group variable was the patient ID. These analyses showed that the difference in serologic decay to all three antigens was dependent upon time only.

PGL-I, phenollic glycolipid-I antigen; R-MDT, regular multidrug therapy; U-MDT, uniform multidrug therapy. positivity. A study among MB Venezuelan patients with high BI showed that the levels of anti-PGL-I antibodies dropped by $57 \%$ over 2 years (30). In general, previous studies have shown $50-90 \%$ drop in anti-PGL-I levels 2 years after conclusion of treatment $(31,46,47)$. Analyses of antibody responses to different antigens in three MB patients showed little, if any, decline in anti-PGL-I serology after MDT (33). Overall, our results show that the gradual drop in antibody levels seen in both U-MDT and R-MDT groups is consistent with a slow reduction in the bacillary load in both treatment groups. In fact, the decline in $\mathrm{BI}$ in $\mathrm{MB}$ patients is known to occur slowly (0.5-1 log U/year), so that a significant proportion of patients with very high $\mathrm{BI}$ at diagnosis, independently of therapy duration, may remain slit skin smear positive for years after treatment (24), therefore stimulating antibody production.

In our study, the percentage of seropositivity varied among tests and also at different time points probably reflecting the sensitivity of the test and the initial bacillary load of the patients. Our data showed that anti-LID-1 serology provided the highest positivity rate at baseline, but as discussed, the decrease in seropositivity was gradual. Among MB patients, the positivity rates remained high 2 years posttreatment, especially to LID-1 
antigen. Our results differ from earlier studies that have shown a faster decline in anti-LID-1 titers compared to anti-PGL-I $(32,33)$. These previous results have suggested that protein antigen was cleared faster than glycolipid/carbohydrate PGL-I antigen. A study showed that a single dose of rifampicin caused a rapid fall in the PGL-I antigen in serum of untreated MB patients (29) and the extent of the reduction of the PGL-I antigen in antibody production is suggested by decreased serology. However, in LL Brazilian patients, the anti-PGL-I antibody levels pre and posttreatment showed a small drop in positivity (from 100 to $90 \%$ ) and also in BL patients (from 100 to 80\%) (32). Results obtained among Venezuelan cured patients who were evaluated 10 years after treatment showed very low antiLID-1 levels (32). Also, similar to our findings, in MB patients with high bacillary load, the anti-LID-1 positivity rates pre and posttreatment were either unchanged or slightly reduced: from 87 to $91 \%$ in LL and from 100 to $79 \%$ in BL patients (32). In another study among Brazilian patients, antibody levels to PGL-I and LID-1 dropped after MDT conclusion, however, some patients remained positive around 2 years after MDT (34). In the current study, non-compliance to MDT can be excluded among the causes for persistent antibody production as all patients were fully compliant to R-MDT and U-MDT; however, the high bacillary burden at diagnosis is compatible with a longer time required for the complete clearance of bacillary antigens. In fact, our results showed that despite the consistent reduction in antibody levels to all three antigens investigated, this decrease was gradual over time, so that 2 years after the conclusion of treatment, a significant percentage of patients continued to be seropositive. In conclusion, for $\mathrm{MB}$ patients with high BI at diagnosis, 2 years follow-up after treatment conclusion seems a short period for a significant clearance of antigens and also for a significant decrease in seropositivity. Therefore, the applicability of serology to monitor treatment efficacy seems limited for MB patients with high bacillary load at diagnosis evaluated in a short-term follow-up after treatment conclusion, such as 2 years.

The loss to follow-up in the first and second year posttreatment represents a limitation of the current study, however, the consistent and gradual decline in antibody levels seen overtime for all three antigens investigated indicates the validity of our data. Our results showed that the decline patterns seen in anti-PGL-I and anti-ND-O-LID serology were very similar. The simultaneous detection of IgM and IgG to the ND-O-LID conjugate which contains the disaccharide epitope of PGL-I and the diffusion LID-1 protein did not enhance sensitivity. A recent study on leprosy patients showed similar high positivity with these three antigens in $\mathrm{BL}$ and $\mathrm{LL}$ patients while the proportion of seropositivity to PGL-I and anti-ND-O-LID antigens was similar but lower than anti-LID-1 positivity (48). Another recent study which evaluated the diagnostic potential of two rapid tests using different antigens (PGL-I and ND-O-LID) and technologies (immunochromatographic lateral flow and luminescent-up-converting phosphor UCP-LFA) showed that both tests corresponded to BI but the UCP-LFA showed higher sensitivity (49). The use of ND-OLID conjugate antigen in leprosy serology, which is expected to enhance sensitivity is recent and results reported so far are not conclusive if detection of $\operatorname{IgM}$ and IgG antibodies to the antigens contained in ND-O-LID effectively leads to higher sensitivity than observed using individual antigens and this topic deserves further investigations.

Previous studies have investigated the potential use of leprosy serology as a marker of reactional episodes (50-52). Two previous publications from our group using the U-MDT database and sera bank $(40,41)$ have described the impact of baseline serology on the development of leprosy reactions. The baseline ML flow test results showed limited sensitivity and specificity as prognostic markers for the development of leprosy reactions during subsequent follow-up (40). Also, the analyses of anti-PGL-I, anti-LID-1, anti-ND-O-LID antibodies at diagnosis showed low sensitivity and specificity for predicting reversal reaction while anti-LID-1 serology at diagnosis showed prognostic value for the development of ENL in BI positive patients (41). We acknowledge the importance of the analysis of the impact of reactional episodes on longitudinal serology data, however, these analyses are out of the scope of the current study which focused on the effect of different treatment regimens on leprosy serology to three antigens. Leprosy serology reflects the bacillary load of the patient and several studies have reported that MB patients are more vulnerable to develop leprosy reactions. In this sense, slit skin smears at diagnosis can indicate patients at higher risk of developing leprosy reactions, but these tests are not part of diagnostic routine and are not used to monitor reactions or relapse. Compared to slit skin smears, serology can be considered a simpler test that could indicate the risk for the development of reactions, especially ENL.

In conclusion, our study on MB leprosy patients, the majority with high bacillary load at diagnosis, indicated a similar decrease in M. leprae antibody production to PGL-I, LID-1, and ND-O-LID in patients treated with R-MDT and U-MDT for 12 and 6 months, respectively. The slow reduction in seropositivity rates seen in MB patients treated with both R-MDT and U-MDT is compatible with the slow decline of bacillary load, regardless of the duration of the treatment. This slow reduction indicates that the applicability of serological monitoring to evaluate MDT efficacy or track the effectiveness of MDT is limited at least in short term period of 2 years posttreatment as within this time period, a significant rate of patients remains positive.

\section{ETHICS STATEMENT}

This study was carried out in accordance with the recommendations of the Resolution 466/2012 from the National Health Council/Ministry of Health, with written informed consent from all subjects. All subjects gave written informed consent in accordance with the Declaration of Helsinki. The protocol was approved by the National Committee for Ethics in Research (CONEP) (protocol number 001/06). Data confidentiality was strictly guaranteed and all patients were free to leave the study and opt for the R-MDT regimen outside the study (ClinicalTrials. gov identifier: NCT00669643). 


\section{AUTHOR CONTRIBUTIONS}

EH, SB-S, RO, GOP, and MMAS conceived and designed the experiments. EH, SB-S, RO, and MMAS performed the experiments. EH, SB-S, MLFP, GOP, and MMAS analyzed the data. SB-S, LA, MAAP, RC, HG, MLFP, GOP, and MMAS contributed with reagents/materials/analysis tools. EH, SB-S, and MMAS wrote the paper. EH, SB-S, and MLFP were in charge of data bank preparation and quality control of data. EH, SB-S, LA, RO, MAAP, HG, MLFP, GOP, and MMAS participated in the interpretation of data, critical reading and approval of the final manuscript. We thank Carlos Sarina, FIOCRUZ, Brasilia for providing high resolution figures.

\section{REFERENCES}

1. Ridley DS, Jopling WH. Classification of leprosy according to immunity. A five-group system. Int J Lepr Other Mycobact Dis (1966) 34(3):255-73.

2. Scollard DM, Adams LB, Gillis TP, Krahenbuhl JL, Truman RW, Williams DL. The continuing challenges of leprosy. Clin Microbiol Rev (2006) 19(2):338-81. doi:10.1128/CMR.19.2.338-381.2006

3. Brett SJ, Payne SN, Gigg J, Burgess P, Gigg R. Use of synthetic glycoconjugates containing the Mycobacterium leprae specific and immunodominant epitope of phenolic glycolipid I in the serology of leprosy. Clin Exp Immunol (1986) 64(3):476-83.

4. Izumi S, Fujiwara T, Ikeda M, Nishimura Y, Sugiyama K, Kawatsu K. Novel gelatin particle agglutination test for serodiagnosis of leprosy in the field. J Clin Microbiol (1990) 28(3):525-9.

5. Buhrer-Sekula S, Cunha MG, Ferreira WA, Klatser PR. The use of whole blood in a dipstick assay for detection of antibodies to Mycobacterium leprae: a field evaluation. FEMS Immunol Med Microbiol (1998) 21(3):197-201. doi:10.1111/j.1574-695X.1998.tb01166.x

6. Buhrer-Sekula S, Sarno EN, Oskam L, Koop S, Wichers I, Nery JA, et al. Use of ML dipstick as a tool to classify leprosy patients. Int J Lepr Other Mycobact Dis (2000) 68(4):456-63.

7. Buhrer-Sekula S, Cunha MG, Foss NT, Oskam L, Faber WR, Klatser PR. Dipstick assay to identify leprosy patients who have an increased risk of relapse. Trop Med Int Health (2001) 6(4):317-23. doi:10.1046/j.1365-3156. 2001.00704.x

8. Buhrer-Sekula S, Smits HL, Gussenhoven GC, van Leeuwen J, Amador S, Fujiwara T, et al. Simple and fast lateral flow test for classification of leprosy patients and identification of contacts with high risk of developing leprosy. J Clin Microbiol (2003) 41(5):1991-5. doi:10.1128/JCM.41.5.19911995.2003

9. Buhrer-Sekula S, Illarramendi X, Teles RB, Penna ML, Nery JA, Sales AM, et al. The additional benefit of the ML flow test to classify leprosy patients. Acta Trop (2009) 111(2):172-6. doi:10.1016/j.actatropica.2009.04.009

10. Young DB, Buchanan TM. A serological test for leprosy with a glycolipidspecific for Mycobacterium leprae. Science (1983) 221(4615):1057-9.

11. Buhrer-Sekula S, Visschedijk J, Grossi MA, Dhakal KP, Namadi AU, Klatser PR, et al. The ML flow test as a point of care test for leprosy control programmes: potential effects on classification of leprosy patients. Lepr Rev (2007) 78(1):70-9.

12. Cole ST, Brosch R, Parkhill J, Garnier T, Churcher C, Harris D, et al. Deciphering the biology of Mycobacterium tuberculosis from the complete genome sequence. Nature (1998) 393(6685):537-44. doi:10.1038/31159

13. Cole ST, Eiglmeier K, Parkhill J, James KD, Thomson NR, Wheeler PR, et al. Massive gene decay in the leprosy bacillus. Nature (2001) 409(6823):1007-11. doi: $10.1038 / 35059006$

14. Reece ST, Ireton G, Mohamath R, Guderian J, Goto W, Gelber R, et al. ML0405 and ML2331 are antigens of Mycobacterium leprae with potential for diagnosis of leprosy. Clin Vaccine Immunol (2006) 13(3):333-40. doi:10.1128/ CVI.13.3.333-340.2006

15. Duthie MS, Goto W, Ireton GC, Reece ST, Cardoso LP, Martelli CM, et al. Use of protein antigens for early serological diagnosis of leprosy. Clin Vaccine Immunol (2007) 14(11):1400-8. doi:10.1128/CVI.00299-07

\section{FUNDING}

This study was supported by Department of Science and Technology and National Council for Scientific and Technological Development (CNPq Number 403293/2005-7). The funders had no role in study design, data collection and analysis, decision to publish, or preparation of the manuscript.

\section{SUPPLEMENTARY MATERIAL}

The Supplementary Material for this article can be found online at https://www.frontiersin.org/articles/10.3389/fimmu.2018.00915/ full\#supplementary-material.

16. Duthie MS, Goto W, Ireton GC, Reece ST, Sampaio LH, Grassi AB, et al. Antigen-specific T-cell responses of leprosy patients. Clin Vaccine Immunol (2008) 15(11):1659-65. doi:10.1128/CVI.00234-08

17. Sampaio LH, Stefani MM, Oliveira RM, Sousa AL, Ireton GC, Reed SG, et al. Immunologically reactive $M$. leprae antigens with relevance to diagnosis and vaccine development. BMC Infect Dis (2011) 11:26. doi:10.1186/1471-2334-11-26

18. Hungria EM, de Oliveira RM, de Souza AL, Costa MB, de Souza VN, Silva EA, et al. Seroreactivity to new Mycobacterium leprae protein antigens in different leprosy-endemic regions in Brazil. Mem Inst Oswaldo Cruz (2012) 107(Suppl 1):104-11. doi:10.1590/S0074-02762012000900017

19. Cardoso LPV, Dias RF, Freitas AA, Hungria EM, Oliveira RM, Collovati M, et al. Development of a quantitative rapid diagnostic test for multibacillary leprosy using smart phone technology. BMC Infect Dis (2013) 13:497. doi:10.1186/1471-2334-13-497

20. Duthie MS, Balagon MF, Maghanoy A, Orcullo FM, Cang M, Dias RF, et al. Rapid quantitative serological test for detection of infection with Mycobacterium leprae, the causative agent of leprosy. J Clin Microbiol (2014) 52(2):613-9. doi:10.1128/JCM.02085-13

21. Duthie MS, Raychaudhuri R, Tutterrow YL, Misquith A, Bowman J, Casey A, et al. A rapid ELISA for the diagnosis of MB leprosy based on complementary detection of antibodies against a novel protein-glycolipid conjugate. Diagn Microbiol Infect Dis (2014) 79(2):233-9. doi:10.1016/j. diagmicrobio.2014.02.006

22. Amorim FM, Nobre ML, Ferreira LC, Nascimento LS, Miranda AM, Monteiro GR, et al. Identifying leprosy and those at risk of developing leprosy by detection of antibodies against LID-1 and LID-NDO. PLoS Negl Trop Dis (2016) 10(9):e0004934. doi:10.1371/journal.pntd.0004934

23. Fabri C, Carvalho AP, Vieira NF, Bueno Ide C, Rodrigues RN, Monteiro TB, et al. Integrative literature review of the reported uses of serological tests in leprosy management. Rev Soc Bras Med Trop (2016) 49(2):158-64. doi:10.1590/ 0037-8682-0226-2015S0037-86822016000200158

24. WHO. 1. Leprosy-Epidemiology. 2. Leprosy-Prevention and Control. 3. LeprosyTherapy. E.C.o.l.e. Report, editor. Geneva, Switzerland: WHO Technical Report Series (2010).

25. Douglas JT. Progress in the serology of leprosy at the University of Hawaii. Hawaii Med J (1988) 47(2):62-5.

26. Chanteau S, Cartel JL, Celerier P, Plichart R, Desforges S, Roux J. PGL-I antigen and antibody detection in leprosy patients: evolution under chemotherapy. Int J Lepr Other Mycobact Dis (1989) 57(4):735-43.

27. Gelber RH, Li F, Cho SN, Byrd S, Rajagopalan K, Brennan PJ. Serum antibodies to defined carbohydrate antigens during the course of treated leprosy. Int J Lepr Other Mycobact Dis (1989) 57(4):744-51.

28. Chaturvedi RM, Kartikeyan S. Employment status of leprosy patients with deformities in a suburban slum. Indian J Lepr (1990) 62(1):109-12.

29. Roche PW, Britton WJ, Failbus SS, Neupane KD, Theuvenet WJ. Serological monitoring of the response to chemotherapy in leprosy patients. Int J Lepr Other Mycobact Dis (1993) 61(1):35-43.

30. Rada EM, Aranzazu N, Convit J. Immunological reactions to mycobacterial proteins in the spectrum of leprosy. Int J Lepr Other Mycobact Dis (1997) 65(4):497-500.

31. Cho SN, Cellona RV, Villahermosa LG, Fajardo TT Jr, Balagon MV, Abalos RM, et al. Detection of phenolic glycolipid I of Mycobacterium leprae in sera from 
leprosy patients before and after start of multidrug therapy. Clin Vaccine Immunol (2001) 8(1):138-42. doi:10.1128/CDLI.8.1.138-142.2001

32. Duthie MS, Hay MN, Rada EM, Convit J, Ito L, Oyafuso LK, et al. Specific IgG antibody responses may be used to monitor leprosy treatment efficacy and as recurrence prognostic markers. Eur J Clin Microbiol Infect Dis (2011) 30(10):1257-65. doi:10.1007/s10096-011-1221-2

33. Spencer JS, Duthie MS, Geluk A, Balagon MF, Kim HJ, Wheat WH, et al. Identification of serological biomarkers of infection, disease progression and treatment efficacy for leprosy. Mem Inst Oswaldo Cruz (2012) 107(Suppl 1): 79-89. doi:10.1590/S0074-02762012000900014

34. Freitas AA, Oliveira RM, Hungria EM, Cardoso LP, Sousa AL, Costa MB, et al. Alterations to antigen-specific immune responses before and after multidrug therapy of leprosy. Diagn Microbiol Infect Dis (2015) 83(2):154-61. doi:10.1016/j.diagmicrobio.2015.06.021S0732-8893(15)00225-4

35. Gonçalves HS, Pontes MA, Buhrer-Sekula S, Cruz R, Almeida PC, Moraes ME, et al. Brazilian clinical trial of uniform multidrug therapy for leprosy patients: the correlation between clinical disease types and adverse effects. Mem Inst Oswaldo Cruz (2012) 107(Suppl 1):74-8. doi:10.1590/S007402762012000900013

36. Penna GO, Pontes MA, Cruz R, Goncalves Hde S, Penna ML, Buhrer-Sekula S. A clinical trial for uniform multidrug therapy for leprosy patients in Brazil: rationale and design. Mem Inst Oswaldo Cruz (2012) 107(Suppl 1):22-7. doi:10.1590/S0074-02762012000900005

37. Penna ML, Buhrer-Sekula S, Pontes MA, Cruz R, Goncalves Hde S, Penna GO. Primary results of clinical trial for uniform multidrug therapy for leprosy patients in Brazil (U-MDT/CT-BR): reactions frequency in multibacillary patients. Lepr Rev (2012) 83(3):308-19.

38. Ferreira IP, Buhrer-Sekula S, De Oliveira MR, Goncalves Hde S, Pontes MA, Penna ML, et al. Patient profile and treatment satisfaction of Brazilian leprosy patients in a clinical trial of uniform six-month multidrug therapy (U-MDT/ CT-BR). Lepr Rev (2014) 85(4):267-74.

39. Penna ML, Buhrer-Sekula S, Pontes MA, Cruz R, Goncalves Hde S, Penna GO. Results from the clinical trial of uniform multidrug therapy for leprosy patients in Brazil (U-MDT/CT-BR): decrease in bacteriological index. Lepr $\operatorname{Rev}(2014)$ 85(4):262-6.

40. Hungria EM, Oliveira RM, Penna GO, Aderaldo LC, Pontes MA, Cruz R, et al. Can baseline ML flow test results predict leprosy reactions? An investigation in a cohort of patients enrolled in the uniform multidrug therapy clinical trial for leprosy patients in Brazil. Infect Dis Poverty (2016) 5(1):110. doi:10.1186/s40249-016-0203-010.1186/s40249-016-0203-0

41. Hungria EM, Buhrer-Sekula S, de Oliveira RM, Aderaldo LC, Pontes AA, Cruz R, et al. Leprosy reactions: the predictive value of Mycobacterium leprae-specific serology evaluated in a Brazilian cohort of leprosy patients (U-MDT/CT-BR). PLoS Negl Trop Dis (2017) 11(2):e0005396. doi:10.1371/ journal.pntd.0005396

42. Stefani MMA, Avanzi C, Buhrer-Sekula S, Benjak A, Loiseau C, Singh P, et al. Whole genome sequencing distinguishes between relapse and reinfection in recurrent leprosy cases. PLoS Negl Trop Dis (2017) 11(6):e0005598. doi:10.1371/journal.pntd.0005598PNTD-D-17-00193

43. Penna GO, Buhrer-Sekula S, Kerr LRS, Stefani MMA, Rodrigues LC, de Araujo MG, et al. Uniform multidrug therapy for leprosy patients in Brazil (U-MDT/CT-BR): results of an open label, randomized and controlled clinical trial, among multibacillary patients. PLoS Negl Trop Dis (2017) 11(7):e0005725. doi:10.1371/journal.pntd.0005725PNTD-D-17-00069

44. Gelman A, Hill J. Data Analysis Using Regression and Multilevel/Hierarchical Models. New York: Cambridge University Press (2007).

45. Kohler U, Kreuter F. Data Analysis Using Stata. College Station, Texas: Stata Press (2009).

46. Silva EA, Iyer A, Ura S, Lauris JR, Naafs B, Das PK, et al. Utility of measuring serum levels of anti-PGL-I antibody, neopterin and C-reactive protein in monitoring leprosy patients during multi-drug treatment and reactions. Trop Med Int Health (2007) 12(12):1450-8. doi:10.1111/j.1365-3156.2007. 01951.x

47. Silva RC, Lyon S, Araos R, Lyon AC, Grossi MA, Lyon SH, et al. The result patterns of ML flow and ELISA (PGL-I) serologic tests in leprosy-endemic and non-endemic areas. Rev Soc Bras Med Trop (2008) 41(Suppl 2):19-22. doi:10.1590/S0037-86822008000700005

48. Fabri ACOC, Carvalho AP, Araujo S, Goulart LR, de Mattos AM, Teixeira $\mathrm{HC}$, et al. Antigen-specific assessment of the immunological status of various groups in a leprosy endemic region. BMC Infect Dis (2015) 15:218. doi:10.1186/ s12879-015-0962-410.1186/s12879-015-0962-4

49. van Hooij A, Tjon Kon Fat EM, van den Eeden SJF, Wilson L, Batista da Silva M, Salgado CG, et al. Field-friendly serological tests for determination of M. leprae-specific antibodies. Sci Rep (2017) 7(1):8868. doi:10.1038/ s41598-017-07803-710.1038/s41598-017-07803-7

50. Stefani MM, Martelli CM, Morais-Neto OL, Martelli P, Costa MB, de Andrade AL. Assessment of anti-PGL-I as a prognostic marker of leprosy reaction. Int $J$ Lepr Other Mycobact Dis (1998) 66(3):356-64.

51. Mizoguti DF, Hungria EM, Freitas AA, Oliveira RM, Cardoso LP, Costa MB, et al. Multibacillary leprosy patients with high and persistent serum antibodies to leprosy IDRI diagnostic-1/LID-1: higher susceptibility to develop type 2 reactions. Mem Inst Oswaldo Cruz (2015) 110(7):914-20. doi:10.1590/ 0074-02760150198

52. Serrano-Coll H, Munoz M, Camilo Beltran J, Duthie MS, Cardona-Castro N. Anti-natural octyl disaccharide-leprosy IDRI diagnostic (NDO-LID) antibodies as indicators of leprosy reactions and neuritis. Trans $R$ Soc Trop Med Hyg (2017) 111(3):125-31. doi:10.1093/trstmh/trx0263867404

Conflict of Interest Statement: The authors declare that the research was conducted in the absence of any commercial or financial relationships that could be construed as a potential conflict of interest.

The reviewer AV declared a shared affiliation with the handling Editor, and the reviewer RP declared a shared affiliation, though no other collaboration, with one of the authors GP.

Copyright (c) 2018 Hungria, Bührer-Sékula, Oliveira, Aderaldo, Pontes, Cruz, Gonçalves, Penna, Penna and Stefani. This is an open-access article distributed under the terms of the Creative Commons Attribution License (CC BY). The use, distribution or reproduction in other forums is permitted, provided the original author(s) and the copyright owner are credited and that the original publication in this journal is cited, in accordance with accepted academic practice. No use, distribution or reproduction is permitted which does not comply with these terms. 